\title{
UK Medical Students' Experience of Culture \& Communication in Cuban Health Care
}

\author{
Jacob Levi MBBS and Isabelle Erbacher MBBS
}

Final-year medical students at UK universities are able to do elective placements elsewhere, to learn about other health care systems. We chose Cuba because of its excellent health outcomes despite relatively few resources. We were struck, for instance, when Cuba made global headlines in 2015 as the first country to eliminate mother-to-child transmission of HIV and syphilis. And, since the UK's underfunded National Health Service is experiencing high demand and increasing pressure, we sought to understand more about Cuba's socialist health system, where health care spending per capita is much lower than the UK's, but life expectancy is almost the same.

In spring 2018, we worked for several weeks at two large obstetric hospitals, one in Havana and another in Santiago de Cuba. While there, we noted differences in doctor-patient and doctor-doctor relationships, compared with the UK (realizing that these are of course subject to cultural norms). Since these were specialized secondary hospitals, we cannot comment on communication norms in primary care settings, where doctors and patients tend to know each other better and have more time to discuss concerns and expectations, and where most women get their antenatal care (with many questions probably answered there before labor even begins).

Communication is fundamental to quality health care. Much of our communication training in the UK focuses on providing patientcentered care, emphasizing provision of clear, understandable information about patients' examinations, diagnosis and treatment. The goal is to facilitate autonomy as well as listening to and answering patients' questions. Obstetrics is a particularly intimate specialty, and pregnancy and childbirth can induce anxiety. In the UK where the doctor-patient ratio is 1:357 (one of the poorest in Europe,[1]) doctors are often unable to dedicate enough time to individual patients because hospitals are dramatically understaffed.

We wondered if Cuba's 1:125 doctor-patient ratio[2] would give doctors more time for communication, but observed that patients often neither expected nor were given copious amounts of information, and instead trusted their doctors to act in their best interests. We witnessed situations where laboring or miscarrying patients were frightened and had questions. Often, they got the information they sought-evidence shows that patients benefit from being involved in treatment decisions and require sufficient information for informed consent-[3] but it was delivered in a very different style from that in the UK. For instance, when a young woman presented with severe abdominal pain in her ninth month and was diagnosed with placental abruption (a dangerous obstetric emergency) she was given only minimal explanation before being rushed to the OR for an emergency C-section; both mother and child were in good health following the birth. It was apparent that she trusted hospital staff to make the right decisions and do what was necessary to deliver her child safely; she did not demand more information from doctors and nurses.

Another contrast between the UK and Cuba is the notion of privacy, possibly due to differing space and resource availability, or to different cultural norms and expectations. The ob-gyn history involves intimate questions about the patient's sexual life, and we observed that, unlike in the UK, Cuban patients often chose to see doctors with a large support network of family members who remained present throughout the consultation, and did not seem to mind if others were present. In Cuban ob-gyn emergency departments in particular, multiple patients sometimes occupied the same room-even sometimes undergoing intimate examinations with relatives present, or even with other nonmedical hospital workers in the room. In contrast, breaching the UK's strict privacy and confidentiality rules can have serious consequences for doctors. UK patients would not tolerate this lack of privacy, but Cuban patients did not seem to feel uncomfortable about it. We also observed that there is less of a taboo around sexuality in Cuba, and people discuss their sexual histories more openly.

The communication among doctors was in some ways more open and honest than we were accustomed to in the UK, where a hierarchy among health care providers can make it difficult (and daunting) for juniors to speak up if they have questions, or importantly, if they think a senior is making a mistake. A hostile work environment can be dangerous, impedes junior doctors from taking responsibility and denies them valuable learning opportunities. The interactions we witnessed between Cuban doctors were respectful, and juniors' concerns were listened to and taken seriously. Such a supportive environment fosters open and honest communication, comradeship and ultimately, we feel, improves patient safety.

It took some time for us to learn, accept and become comfortable with these different cultural norms, before we were able to participate fully in Cuban hospitals. Once we did, however, we found that health care staff were exceptional teachers and keen to share their knowledge with juniors. We learned a great deal about these cultural differences, especially after discussing them with Cuban doctors who had had medical placements abroad.

In medicine, as in many aspects of life, learning to appreciate and understand cultural differences can lead to mutual benefits and new ideas. Our Cuban colleagues and patients must have had similarly bewildered thoughts about our culture and behavior. Our experiences in Cuba helped reinforce the importance of knowledge sharing and understanding the diversity of attitudes and styles in health care provision. $-1 /$ -

1. Moberly T. UK has fewer doctors per person than most other OECD countries. BMJ [Internet]. 2017 [cited 2018 Oct 10];357:j2940. Available from: http:// careers.bmj.com/careers/advice/UK_has_fewer_doctors_per_person_than _most_other_OECD_countries

2. National Health Statistics and Medical Records Division (CU). Anuario Estadístico de Salud 2016 [Internet]. Havana: Ministry of Public Health (CU); 2017 [cited 2018 Oct 10]. 206 p. Available from: http://files.sld.cu/dne/files/2017/05/Anua rio_Estad\%C3\%ADstico_Ingles_e_2016_Edici\%C3\%B3n_2017.pdf. Spanish.

3. Hill $A E$, Smith $C V$, Hadden BW. Autonomy in the obstetrician/gynecologistpatient relationship as a predictor of patient satisfaction. Yale J Biol Med [Internet]. 2013 Jun 13 [cited 2018 Oct 10];86(2):179-88. Available from: https:// www.ncbi.nlm.nih.gov/pubmed/23766739

Submitted July 6, 2018

Approved: October 10, 2018

Disclosures: None

Correspondence: jacob.adam.levi11@gmail.com 